\title{
Improved Directivity of an OAM Antenna by a Fabry-Perot Cavity: An Experimental Study
}

\author{
Wenlong Wei ${ }^{1}$, Wenlin Kuai' ${ }^{1}$, Kourosh Mahdjoubi ${ }^{2}$, Christian Brousseau², Olivier Emile ${ }^{3}$ \\ ${ }^{1}$ Shanghai Satellite Engineering Institute, Shanghai, China \\ ${ }^{2}$ Institute of Electronics and Telecommunications of Rennes (IETR), University of Rennes 1, Rennes, France \\ ${ }^{3}$ Laser Physics Laboratory (LPL), University of Rennes 1, Rennes, France \\ Email:wwl880914@163.com
}

How to cite this paper: Wei, W.L., Kuai, W.L., Mahdjoubi, K., Brousseau, C. and Emile, O. (2021) Improved Directivity of an OAM Antenna by a Fabry-Perot Cavity: An Experimental Study. Open Journal of Antennas and Propagation, 9, 65-73.

https://doi.org/10.4236/ojapr.2021.94006

Received: October 21, 2021

Accepted: December 25, 2021

Published: December 28, 2021

Copyright (C) 2021 by author(s) and Scientific Research Publishing Inc. This work is licensed under the Creative Commons Attribution International License (CC BY 4.0).

http://creativecommons.org/licenses/by/4.0/

\begin{abstract}
The circular phased antenna array is commonly used for generating waves bearing Orbital Angular Momentum (OAM) in the radio frequency band, but it achieves a relatively low directivity. To overcome this drawback, we present here a method to improve the directivity of an OAM circular phased antenna array by embedding it inside a Fabry-Perot cavity. The Fabry-Perot cavity contains three main parts: a partially reflecting surface (PRS), an air cavity and a ground plane. Simulation data show that the directivity of this new OAM antenna achieves an improvement of $8.2 \mathrm{~dB}$ over the original array. A prototype is realized and characterized. The simulated and measured characteristics are in good agreement.
\end{abstract}

\section{Keywords}

Circular Antenna Array, Fabry-Perot Cavity, Orbital Angular Momentum, Patch Antenna, Phase Shifter

\section{Introduction}

Orbital angular momentum (OAM) has been proposed to improve spectral efficiency [1] [2] [3] [4] in radio communications, by creating multiple sub-channels of propagation corresponding to the twisting degree of the electromagnetic wave. Several applications for object identification [5] and radars [6] [7] have also been proposed.

Whereas the phase of a usual plane wave is constant on the wavefront, the phase $\alpha$ of OAM waves undergoes a linear variation along the angular coordinate $\varphi$ (roll angle): $\alpha=I \varphi$, where $l$ is an integer number called the "topological charge" or the order of the OAM mode. 
At radio frequencies, a single patch antenna [8] [9] [10] [11] or a phased array of patch antennas [11] [12] [13] is proposed to generate the phase variation of waves bearing OAM. However, the poor directivity of the OAM radiation can lead to some drawbacks and limitations, especially in terms of link budget [14]. On the other hand, a second family of antennas can be found in the literature, where the directivity is quite high. As an example, we can mention the reflector antenna using an $80 \mathrm{~cm}$ twisted parabolic reflector dish to induce a linear phase distribution (along the $\varphi$ angle) at a working frequency of $2.4 \mathrm{GHz}$ [2]. Later, spiral phase plates (SPP) [15] [16] and flat drilled phase plates [17] [18] have been used to obtain the linear phase variation in the millimeter wave frequency band. The OAM dish needs precise deformation of the reflector shape to ensure the linear variation of the phase $\alpha$. However, these deformations create new aberrations due to the fact that no focal point can be defined for these reflectors. Concerning the structures that use dielectric lenses (SPP or flat drilled ones), the plates are heavy and at microwave frequencies they become unpractical.

In previous work, a simple antenna array has been embedded inside a FabryPerot cavity to obtain a relatively high directivity at simulation level [19]. Here, we make an experimental study of this high directivity OAM antenna. To the purpose of realization, we embed an OAM antenna, using four patches and a circular phase shifter inside a Fabry-Perot cavity. This antenna generates an electromagnetic wave bearing an OAM mode of $l=1$ at the frequency of 2.5 $\mathrm{GHz}$.

Section 2 and 3 explain the design procedure and the simulation data. Section 4 presents the experimental prototype and the measured characteristics of the antenna.

\section{Model of Fabry-Perot Cavity}

A Fabry-Perot (FP) cavity was originally used as frequency filter in optics. In antenna applications, it is often utilized as space filter to improve the antenna performances such as directivity [20] [21] [22].

As shown in Figure 1, the FP cavity contains three main parts: a ground plane which eliminates the back radiation, a primary source (e.g. a patch antenna) and a partially reflecting surface (PRS). The internal wave rays emitted by the primary source travel inside the cavity and reflect for enough times at both the upper PRS and the bottom ground plane. Besides, the wave rays partially transmit out when they arrive at the PRS each time.

To maximize the directivity, the transmitted rays need to all be in phase so that they can make a constructive interference. According to [17], the thickness $D$ of the FP cavity should meet the following requirement:

$$
D=\frac{c}{4 \pi f_{0} \cos \theta}[\beta+(2 n+1) \pi]
$$

where $f_{0}$ is the working frequency, $\beta$ the phase of the reflection coefficient of the PRS, $c$ the speed of the light in the air cavity, $n$ an integer number corresponding 
to the cavity mode, and $\theta$ the incidence angle of the rays (see Figure 1 ).

\section{Antenna Design and Simulation Results}

The geometry of the Fabry-Perot OAM antenna is shown in Figure 2. The exciting OAM source consists of an array of 4 patches and a circular phase shifter, working at $2.5 \mathrm{GHz}$. The array radius is $60 \mathrm{~mm}$ and the patch antennas and phase shifter are fabricated on a FR4 substrate with a thickness of $1.6 \mathrm{~mm}$ and a relative permittivity of 4.4 . The patch antennas and the phase shifter are connected through probes and the phase shifter is fed by a $50 \Omega$ coaxial cable from

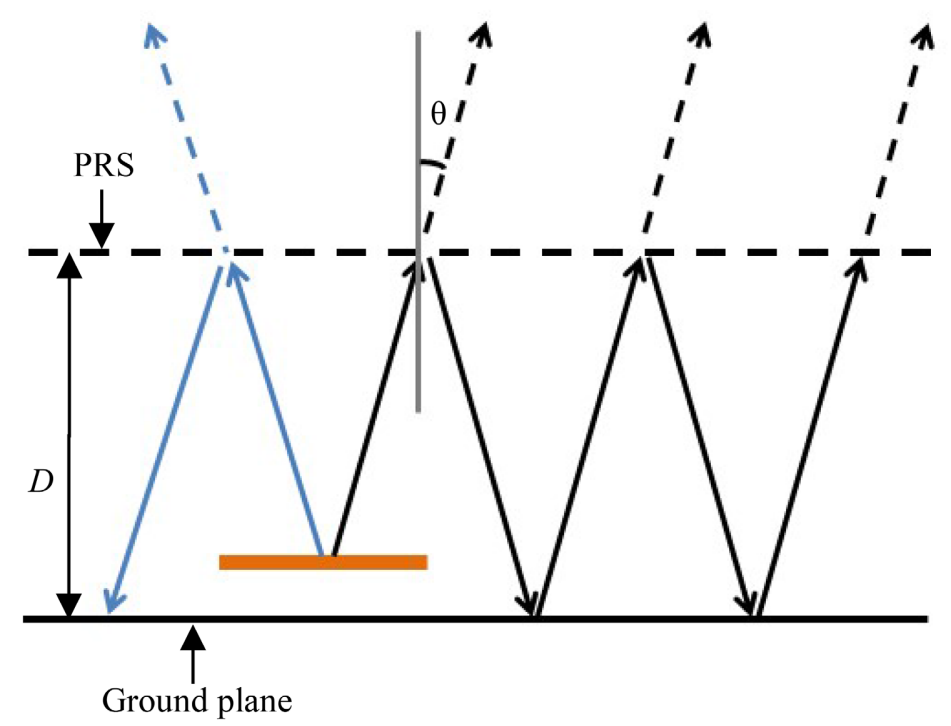

Figure 1. Model of the FP cavity with PRS (PRS: Partially reflecting surface).

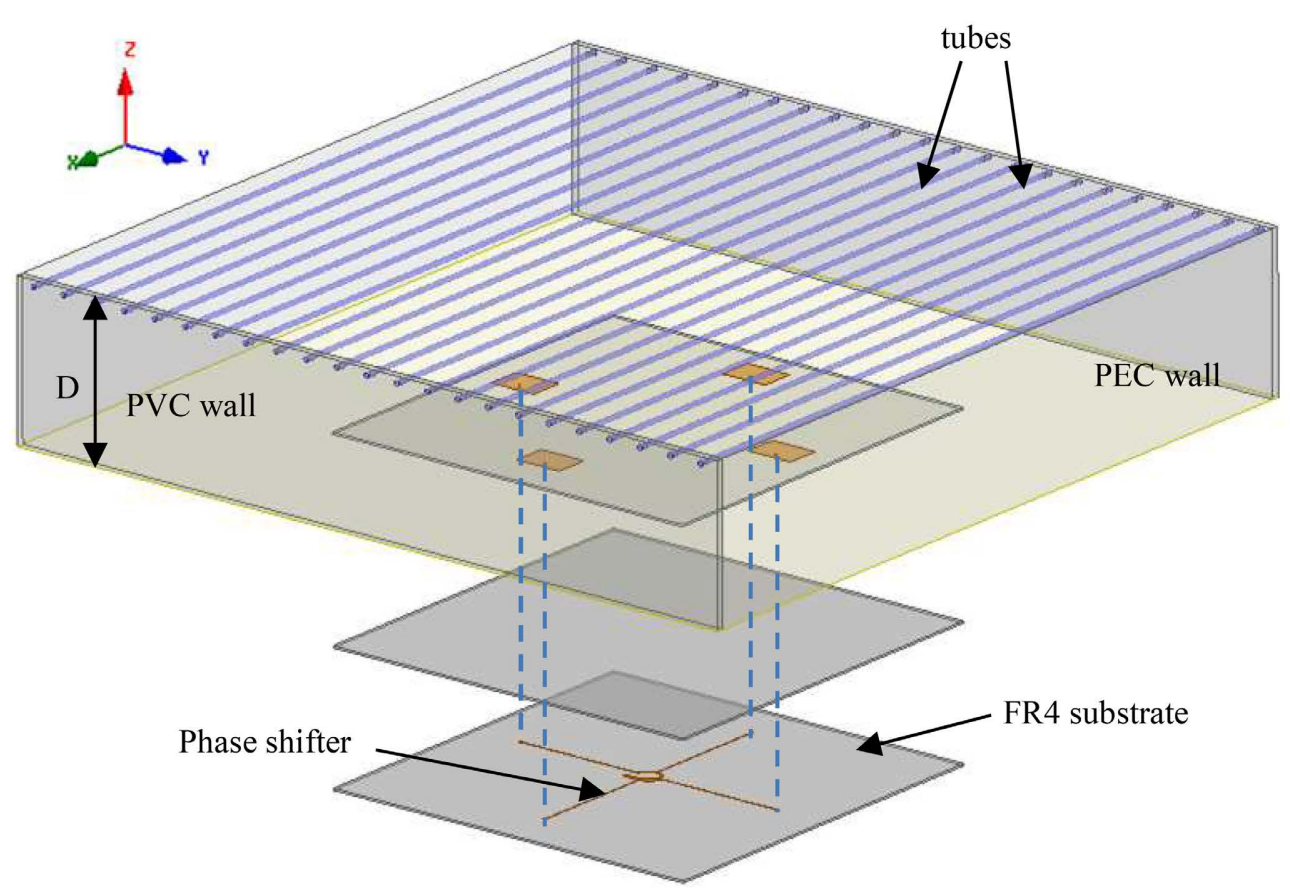

Figure 2. Geometry of the Fabry-Perot OAM antenna. 
the bottom side of the substrate. To generate radio waves bearing an OAM mode of $l=1$, the length and width of the microstrip feeding lines are optimized to ensure the antenna matching, a uniform amplitude for all the array elements and a $90^{\circ}$ phase shift between two successive elements [13].

The directivity of the Fabry-Perot cavity itself depends on the thickness $D$, the reflectivity and the area of the PRS [20]. In our design, the PRS is made of metallic tubes oriented in parallel to the $\mathrm{E}$ field of the patch antennas (X axis). To obtain a good directivity of the OAM antenna, the PRS and the cavity parameters have been optimized using the HFSS software. The following values are obtained:

- Diameter of the tubes d: $4 \mathrm{~mm}$;

- Period of the tubes T: $26 \mathrm{~mm}$;

- Cavity thickness D: $58 \mathrm{~mm}$;

- Cavity aperture: $600 \mathrm{~mm} \times 600 \mathrm{~mm}$.

Furthermore, we use 4 walls to enclose the FP cavity on the sides: 2 PEC (Perfect electric conductor) walls in the E plane and 2 PVC (Polyvinyl Chloride) walls in the $\mathrm{H}$ plane. The PEC walls are used to decrease the side lobe level of the radiation pattern in the $\mathrm{H}$ plane. The PVC walls support the metallic tubes. According to the simulations, in order to decrease the side lobe level in the E plane, the thickness of the PVC wall must be small enough. On the other hand, the PVC walls should be allowed to assemble properly with the PEC walls and the ground plane, and to support the metallic tubes. To meet these requirements, the thickness of the PVC walls is finally set as $6 \mathrm{~mm}$.

Figure 3 shows the 2D simulated amplitude and phase patterns of the $E_{x}$ component of the generated wave at $2.5 \mathrm{GHz}$. The patterns are plotted on a plane perpendicular to the direction of propagation with an area of $40 \mathrm{~cm} \times 40$ $\mathrm{cm}$, lying $3 \mathrm{~cm}$ above the PRS. We can see that the amplitude at the centre is much weaker than the surroundings and the phase makes a rotation around the centre with a $2 \pi$ phase shift in one turn. These characteristics evidence the generation of an OAM bearing wave with $l=1$, as expected.
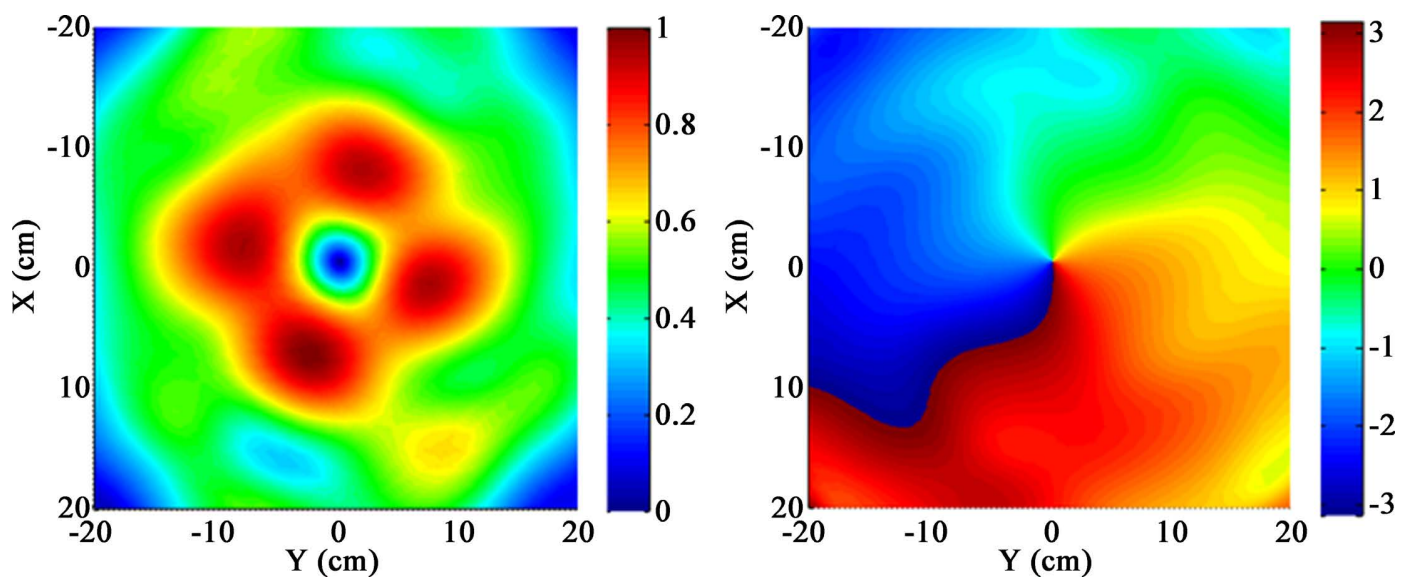

Figure 3. Normalized simulated amplitude (left) and phase (right) patterns of $E_{x}$ component of the FP OAM antenna at $2.5 \mathrm{GHz}$. 
The simulated 3D radiation patterns of the patch array with and without FP cavity, at $2.5 \mathrm{GHz}$, are presented in Figure 4 . The array radius is $60 \mathrm{~mm}$. A null can be observed in the centre which is the typical characteristic of waves bearing OAM. Besides, the antenna directivity is significantly enhanced with the use of the FP cavity. The directivity can be further improved by increasing the radius of the patch array.

In order to exemplify the influence of the FP cavity on the antenna directivity, we make a comparison of the E-plane radiation patterns (Figure 5) corresponding to the $\mathrm{xOz}$ cuts of the 3D plots of Figure 4 . We can see that the directivity of the OAM antenna increases in E plane, from 6.7 to $14.9 \mathrm{~dB}$ with the use of the FP cavity. At the same time, the angle for obtaining the maximum directivity decreases from $27^{\circ}$ to $11^{\circ}$.

\section{Realization and Experiment}

The realized prototype of the Fabry-Perot OAM antenna is shown in Figure 6. The measurements have been performed in an anechoic chamber.

Figure 7 shows the simulated and measured reflection coefficients of the FP

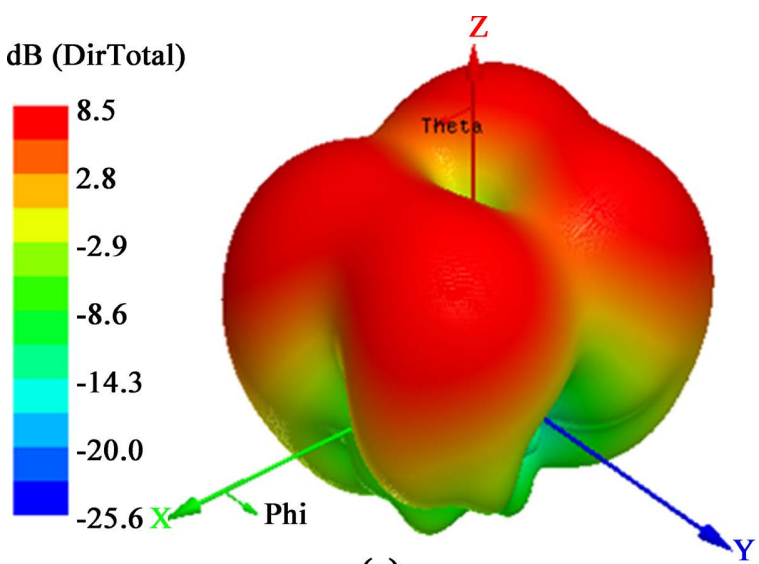

(a)

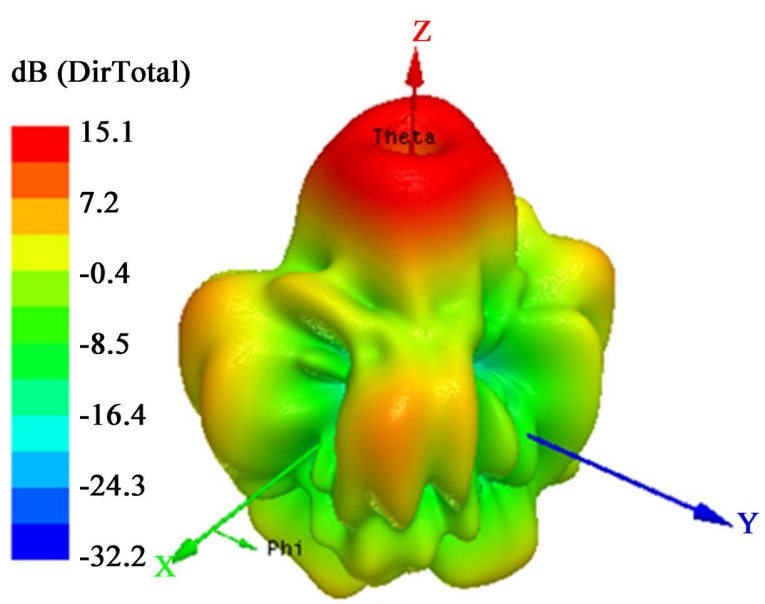

(b)

Figure 4. Simulated 3D radiation patterns of the OAM antenna at $2.5 \mathrm{GHz}$ (a) without FP cavity, (b) with FP cavity. 


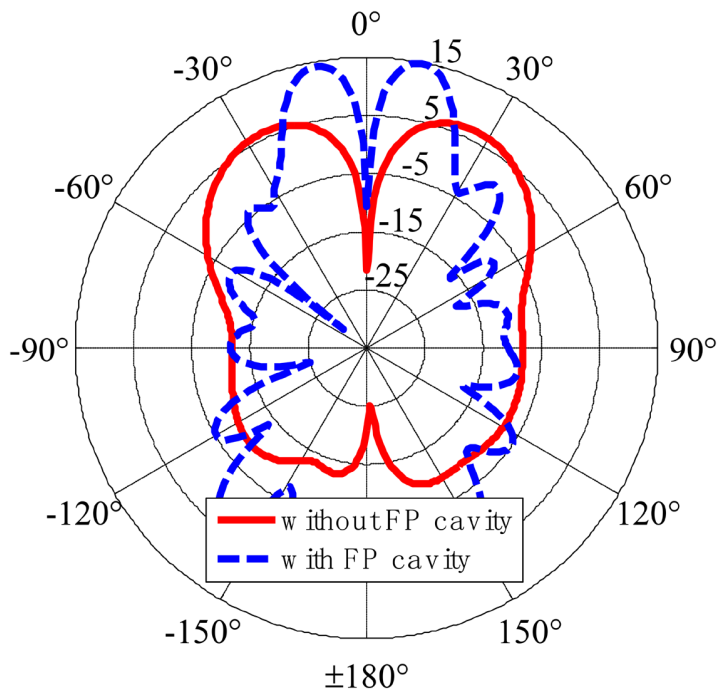

Figure 5. E-plane radiation patterns of the OAM antenna with and without FP cavity (array radius equals to $60 \mathrm{~mm}$ ).

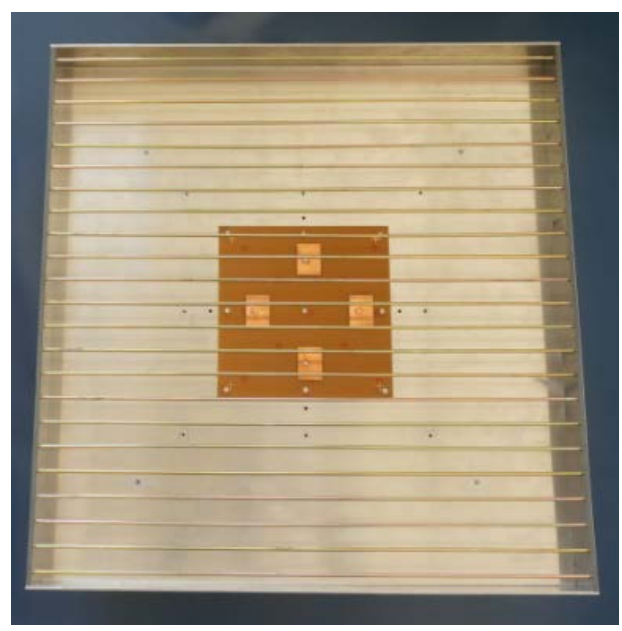

Figure 6. Realized prototype of the FP OAM antenna.

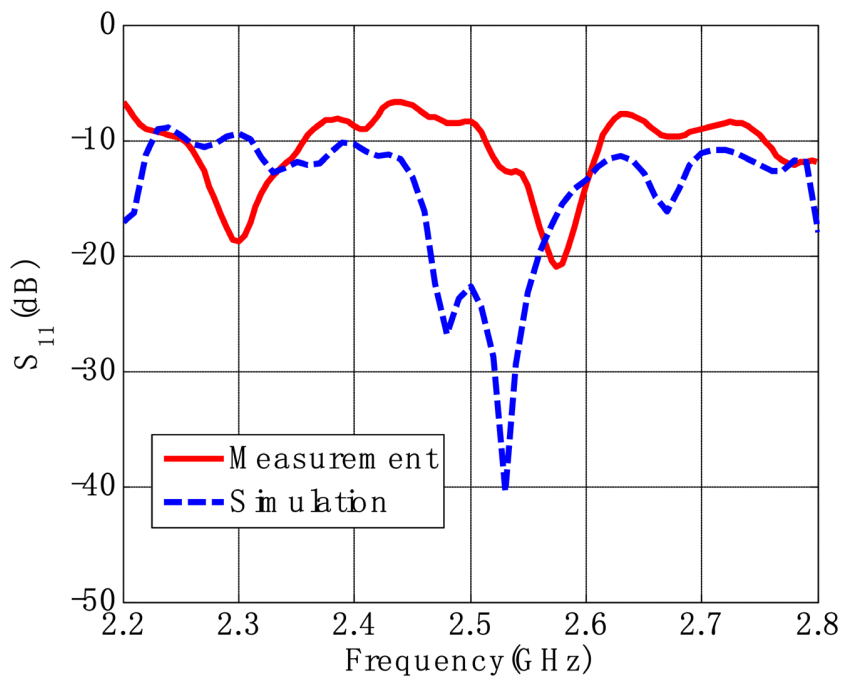

Figure 7. Simulated and measured reflection coefficients of the FP OAM antenna. 
OAM antenna. Whereas the antenna is well matched around $2.5 \mathrm{GHz}$ in the simulation, it is slightly shifted by about $40 \mathrm{MHz}$ in the measurement.

Figure 8 presents the experimental results of the amplitude and phase of the generated far-field. We have examined the radiation results for different frequencies and found that the best OAM characteristic is obtained at $2.54 \mathrm{GHz}$. As shown, a null caused by the phase singularity at the centre, is clearly observed in the magnitude pattern and the phase has a variation of $2 \pi$ in one turn which corresponds to the first OAM mode $(l=1)$.

A comparison is made between the simulated and measured E-plane radiation patterns in Figure 9. As shown, the OAM antenna obtains a maximum directivity of $15.5 \mathrm{~dB}$ for the angle of $10^{\circ}$ in the measurement. Besides, the measured and simulated results are in very good agreement. The hole in the centre of the radiation pattern seems even deeper in the case of the experimental values than for the simulations.

\section{Conclusion}

In this paper, we have proposed a new OAM antenna for the generation of radio
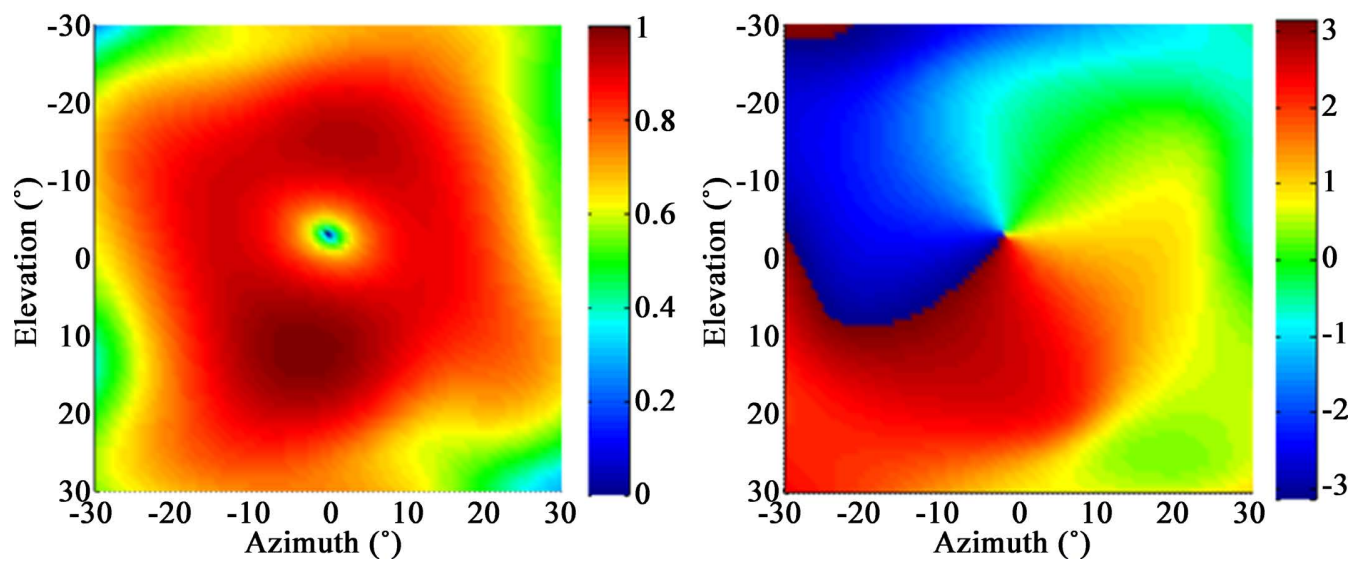

Figure 8. Normalized measured far-field magnitude (left) and phase (right) patterns of the FP OAM antenna at $2.54 \mathrm{GHz}$.

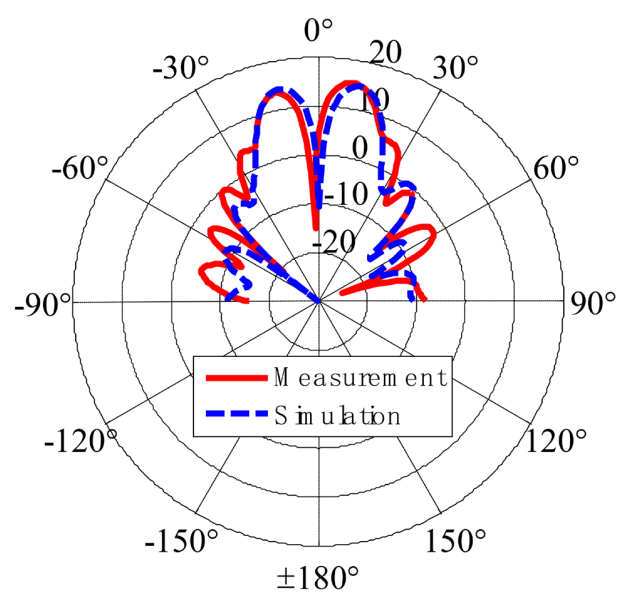

Figure 9. Measured and simulated radiation patterns of the FP OAM antenna in E-plane. 
OAM waves with relatively high directivity. This OAM antenna is based on an array of 4 patches and is embedded inside a Fabry-Perot cavity. It can generate an electromagnetic wave bearing an $l=1 \mathrm{OAM}$ mode at $2.5 \mathrm{GHz}$. Simulation results show that the OAM antenna achieves a directivity of $14.9 \mathrm{~dB}$ with an enhancement of $8.2 \mathrm{~dB}$ in $\mathrm{E}$ and $\mathrm{H}$ planes. A prototype has been manufactured and characterized. Very good agreement is obtained between the simulated and measured directivities. The structure of the new OAM antenna is simple, compact and easy to realize. It can be used in many domains such as radio communications and radar applications.

\section{Conflicts of Interest}

The authors declare no conflicts of interest regarding the publication of this paper.

\section{References}

[1] Mohammadi, S.M., Daldorff, L., Bergman, J., et al. (2010) Orbital Angular Momentum in Radio-A System Study. IEEE Transactions on Antennas and Propagation, 58, 565-572. https://doi.org/10.1109/TAP.2009.2037701

[2] Tamburini, F., Mari, E., Sponselli, A., et al. (2012) Encoding Many Channels on the Same Frequency through Radio Vorticity: First Experimental Test. New Journal of Physics, 14, Article ID: 033001. https://doi.org/10.1088/1367-2630/14/3/033001

[3] Wang, J., Yang, J.Y., Ahmed, N., et al. (2012) Terabit Free-Space Data Transmission Employing Orbital Angular Momentum Multiplexing. Nature Photonics, 6, 488-496. https://doi.org/10.1038/nphoton.2012.138

[4] Yan, Y., Xie, G.D., Ahmed, N., et al. (2014) High-Capacity Millimetre-Wave Communications with Orbital Angular Momentum Multiplexing. Nature Communications, 5, Article No. 4876. https://doi.org/10.1038/ncomms5876

[5] Patarroyo, N.U., Fraine, A., Simon, D.S., et al. (2013) Object Identification Using Correlated Orbital Angular Momentum States. Physical Review Letters, 110, 043601. https://doi.org/10.1103/PhysRevLett.110.043601

[6] Liu, K., Chen, Y.Q., Yang, Z.C., et al. (2015) Orbital-Angular-Momentum-Based Electromagnetic Vortex Imaging. IEEE Antennas and Wireless Propagation Letters, 14, 711-714. https://doi.org/10.1109/LAWP.2014.2376970

[7] Lin, M., Gao, Y., Liu, P., et al. (2016) Super-Resolution Orbital Angular Momentum Based Radar Targets Detection. Electronics Letters, 52, 1168-1170.

https://doi.org/10.1049/el.2016.0237

[8] Barbuto, M., Trotta, F., Bilotti, F., et al. (2014) Circular Polarized Patch Antenna Generating Orbital Angular Momentum. Progress in Electromagnetic Research, 148, 23-30. https://doi.org/10.2528/PIER14050204

[9] Singh, S., Upadhayay, M.D., Pal, S., OAM (2020) Wave Generation Using SquareShaped Patch Antenna as Slot Array Equivalence. IEEE Antennas and Wireless Propagation Letters, 19, 680-684. https://doi.org/10.1109/LAWP.2020.2976611

[10] Li, W.W., Zhu, J.B., Liu, Y.C., et al. (2020) Realization of Third Order OAM Mode Using Ring Patch Antenna. IEEE Transactions on Antennas and Propagation, 68, 7607-7611. https://doi.org/10.1109/TAP.2020.2990311

[11] Guo, C., Zhao, X.W., Zhu, C., et al. (2019) An OAM Patch Antenna Design and Its 
Array for Higher Order OAM Mode Generation. IEEE Antennas and Wireless Propagation Letters, 18, 816-820. https://doi.org/10.1109/LAWP.2019.2900265

[12] Bai, Q., Tennant, A., and Allen, B. (2014) Experimental Circular Phased Array for Generating OAM Radio Beams. Electronics Letters, 50, 1414-1415.

https://doi.org/10.1049/el.2014.2860

[13] Wei, W.L., Mahdjoubi, K., Brousseau, C., Emile, O. (2015) Generation of OAM Waves with Circular Phase Shifter and Array of Patch Antennas. Electronics Letters, 51, 442-443. https://doi.org/10.1049/el.2014.4425

[14] Nguyen, D. K., Pascal, O., Sokoloff, J., et al. (2015) Antenna Gain and Link Budget for Waves Carrying Orbital Angular Momentum. Radio Science, 50, 1165-1175. https://doi.org/10.1002/2015RS005772

[15] Turnbull, G.A., Robertson, D.A., Smith, G.M., et al. (1996) The Generation of FreeSpace Laguerre-Gaussian Modes at Millimeter-Wave Frequencies by Use of a Spiral Phaseplate. Optics Communications, 127, 183-188. https://doi.org/10.1016/0030-4018(96)00070-3

[16] Hui, X., Zheng. S., Hu. Y., et al. (2015) Ultralow Reflectivity Spiral Phase Plate for Generation of Millimeter-Wave OAM beam. IEEE Antennas and Wireless Propagation Letters, 14, 966-969. https://doi.org/10.1109/LAWP.2014.2387431

[17] Niemiec, R., Brousseau, C., Mahdjoubi, K., Emile, O. (2014) Characterization of an OAM flat Plate Antenna in the Millimeter Frequency Band. IEEE Antennas and Wireless Propagation Letters, 13, 1011-1014. https://doi.org/10.1109/LAWP.2014.2326525

[18] Cheng, L., Hong, W., and Hao, Z.C. (2014) Generation of Electromagnetic Waves with Arbitrary Orbital Angular Momentum Modes. Scientific Reports, 4, Article No. 4814. https://doi.org/10.1038/srep04814

[19] Wei, W.L., Mahdjoubi, K., Brousseau, C., et al. (2016) Enhancement of Directivity of an OAM Antenna by Using Fabry-Perot Cavity. 10th European Conference on Antennas and Propagation (EuCAP), Davos, Switzerland, 10-15 April 2016, 1-4. https://doi.org/10.1109/EuCAP.2016.7481906

[20] Mahdjoubi, K., Vu, T.H., Tarot, A.C., et al. (2010) An Overview on the Design and Properties of EBG Antennas. Proceedings of the Fourth European Conference on Antennas and Propagation, Barcelona, Spain, 12-16 April 2010, 1-3.

[21] Akalin, T., Danglot, J., Vanbésien, O., et al. (2002) A Highly Directive Dipole Antenna Embedded in a Fabry-Perot Type Cavity. IEEE Microwave and Wireless Components Letters, 12, 48-50. https://doi.org/10.1109/7260.982873

[22] Boutayeb, H., Mahdjoubi, K., Tarot, A.C., et al. (2006) Directivity of an Antenna Embedded Inside a Fabry-Perot Cavity: Analysis and Design. Microwave and Optical Technology Letters, 48, 12-17. https://doi.org/10.1002/mop.21249 